\title{
Orbifold matrix models and fuzzy extra dimensions
}

\author{
Athanasios Chatzistavrakidis* \\ Bethe Center for Theoretical Physics and Physikalisches Institut, University of Bonn \\ Nussallee 12, D-53115 Bonn, Germany \\ E-mail: than@th.physik.uni-bonn.de
}

Harold Steinacker

Faculty of Physics, University of Vienna

Boltzmanngasse 5, A-1090 Vienna, Austria

and

Physics Department

City College of the City University of New York

160 Convent Avenue, New York, NY 10031

E-mail: harold.steinacker@univie.ac.at

\section{George Zoupanos ${ }^{\dagger}$}

Theory Group, Physics Department

CERN, Geneva, Switzerland

E-mail: George.Zoupanosecern.ch

\begin{abstract}
We revisit an orbifold matrix model obtained as a restriction of the type IIB matrix model on a $\mathbb{Z}_{3}$-invariant sector. An investigation of its moduli space of vacua is performed and issues related to chiral gauge theory and gravity are discussed. Modifications of the orbifolded model triggered by Chern-Simons or mass deformations are also analyzed. Certain vacua of the modified models exhibit higher-dimensional behaviour with internal geometries related to fuzzy spheres.
\end{abstract}

Proceedings of the Corfu Summer Institute 2011 School and Workshops on Elementary Particle Physics and Gravity

September 4-18, 2011

Corfu, Greece

Preprint number: CERN-PH-TH/2012-057

\footnotetext{
* Speaker.

${ }^{\dagger}$ On leave from Physics Department, National Technical University, Zografou Campus: Heroon Polytechniou 9, 15780 Zografou, Athens, Greece
} 


\section{Introduction}

The theoretical efforts to establish a deeper understanding of Nature has led to a huge and sustained research activity in two very interesting frameworks, namely Superstring Theories [1] and Non-Commutative Geometry [2]. Superstring Theory is often regarded as the best candidate for a quantum theory of gravitation, or more generally as a unified theory of all fundamental interactions. Similarly Non-Commutative space-time and in general Non-Commutative Geometry is expected, on quite general grounds, to be the natural arena to study a quantum theory of gravity. In addition the two frameworks came closer by realizing that in M-Theory and in open String Theory, in the presence of a non-vanishing background antisymmetric field, the effective physics on D-branes can be described by a Non-Commutative gauge theory [3,4]. Thus Non-Commutative field theories emerge as effective description of string dynamics. Moreover the type IIB superstring theory, related to the other superstring theories by certain dualities, in its conjectured non-perturbative formulation as a matrix model [5], is a non-commutative theory. Finally the String Theory and NonCommutative Geometry approaches share similar hopes for exhibiting improved renormalization properties in the ultraviolet regime as compared to ordinary field theories.

Non-commutative geometry has been regarded as a promising framework for constructing finite quantum field theories or at least as a natural scheme for regularizing quantum field theories. However the quantization of field theories on non-commutative spaces has turned out to be much more difficult than expected and with problematic ultraviolet features [6], see however [7], and [8]. Although models of the Standard Model (SM) type have been constructed using the Seiberg-Witten map, they can only be considered as effective theories and they are not renormalizable. A drastic change in the perspective of non-commutative geometry was given with the suggestion that indeed it might be relevant for particle physics models but in the description of extra dimensions [9]. The higher-dimensional theories that can be constructed based on this proposal, appeared to have many interesting unexpected features ranging from their renormalizability to their potential predictivity.

In the context of higher-dimensional gauge theories, we have explored the possibility that the extra dimensions are described by matrix approximations to smooth manifolds known as fuzzy spaces. Initially, higher-dimensional gauge theories defined on the product of Minkowski space and a fuzzy coset space $(S / R)_{F}$ were considered and their dimensional reduction to four dimensions was performed, using the Coset Space Dimensional Reduction scheme (CSDR) [10, 11, 12]. Although for the technicalities one has to consult the original or some review papers [9], a major difference between fuzzy and ordinary CSDR is that the four-dimensional gauge group appearing in the ordinary CSDR after the geometrical breaking and before the spontaneous symmetry breaking due to the four-dimensional Higgs fields does not appear in the fuzzy CSDR. In fuzzy CSDR the spontaneous symmetry breaking takes already place by solving the fuzzy CSDR constraints and the four-dimensional potential appears already shifted to a minimum. Therefore in four dimensions appears only the physical Higgs field that survives after a spontaneous symmetry breaking. Correspondingly in the Yukawa sector of the theory we have the results of the spontaneous symmetry breaking, i.e. massive fermions and Yukawa interactions among fermions and the physical Higgs field. The conclusion is that if one would like to describe the spontaneous symmetry breaking of the SM in this framework, then one would be naturally led to consider large extra dimensions. A fundamental difference between the ordinary CSDR and its fuzzy version is the fact that a non-Abelian 
gauge group $\mathrm{G}$ is not really required in many dimensions. Indeed it turns out that the presence of a $U(1)$ in the higher-dimensional theory is enough to obtain non-Abelian gauge theories in four dimensions.

Another fundamental difference as compared to all known theories defined in more than four dimensions is that the present ones are renormalizable. Arguments were given supporting this observation [9] but the strongest support came by studying the whole problem from another point of view. Specifically, in a further important development we presented a renormalizable fourdimensional $S U(N)$ gauge theory with a suitable multiplet of scalars, which dynamically develops fuzzy extra dimensions that form a fuzzy sphere [13]. This model has non-trivial vacua which admit an interpretation as 6-dimensional gauge theory, with gauge group and geometry depending on the parameters appearing in the original Lagrangian. A finite tower of massive Kaluza-Klein modes arises, consistent with an interpretation as compactified higher-dimensional gauge theory. There are many remarkable aspects of this model. First, it provides an extremely simple and geometrical mechanism of dynamically generating extra dimensions. This is based on an important lesson from non-commutative gauge theory, namely that non-commutative or fuzzy spaces can be obtained as solutions of matrix models. The mechanism is quite generic, and does not require fine-tuning or supersymmetry. This provides in particular a realization of the basic ideas of compactification and dimensional reduction within the framework of renormalizable quantum field theory. Moreover, being essentially a large $N$ gauge theory, the analytical techniques developed in this context should be applicable. In particular, it turns out that the generic low-energy gauge group is given by $S U\left(n_{1}\right) \times S U\left(n_{2}\right) \times U(1)$ or $S U(n)$, while gauge groups which are products of more than two simple components (apart from $U(1)$ ) do not seem to occur in this model. Moreover, a magnetic flux is naturally induced in the vacua with non-simple gauge group.

The appealing features of the above mechanism naturally suggest to find models which are phenomenologically relevant to particle physics. One of the most challenging problems in this direction is the accommodation of chiral fermions in four dimensions. The inclusion of fermions in the above class of models showed that the best one could achieve without further requirements is to obtain theories with mirror fermions in bi-fundamental representations of the low energy gauge group [14]. Indeed, studying in detail the fermionic sector of models which dynamically develop extra dimensions with the geometry of a fuzzy sphere or a product of two fuzzy spheres we found [15] that the low-energy theory contains two mirror sectors, even when magnetic fluxes are included on the two fuzzy spheres. Although mirror fermions do not exclude the possibility to make contact with phenomenology [16], it is clearly desirable to obtain exactly chiral fermions. This was achieved by introducing an additional structure in the above context, based on orbifolds. In particular, a $\mathbb{Z}_{3}$ orbifold projection of a $N=4 S U(3 N)$ SYM theory leads to a $N=1$ supersymmetric theory with gauge group $S U(N)^{3}$ [17]. Adding a suitable set of soft supersymmetry breaking terms in the $N=1$ theory, certain vacua of the theory were revealed, where twisted fuzzy spheres are dynamically generated. It is well known that the introduction of a soft supersymmetry breaking sector is not only natural but also necessary in the constructions of phenomenologically viable supersymmetric theories, with prime example the case of the MSSM. Such vacua correspond to models which retain the welcome features described above and in addition they exhibit a chiral low-energy spectrum. The potentially most interesting chiral models for low-energy phenomenology which can be constructed in this context turn out to be $S U(4) \times S U(2) \times S U(2), S U(4)^{3}$ and 
$S U(3)^{3}$. Eventually the most interesting case turned out to be the a unified theory based on the trinification group, which can be promoted even to an all-loop finite theory (for a review see [18]) and moreover it is suitable to make testable predictions [19]. Thus it was shown that fuzzy extra dimensions can arise in simple field-theoretical models which are chiral, renormalizable, and maybe phenomenologically viable.

In the present contribution, we would like to report on a natural extension of the above ideas and methods, realized in the context of Matrix Models (MM). MMs offer a framework where both profound conceptual problems as well as questions about low-energy physics may be addressed. Indeed, at a fundamental level, the MMs introduced by Banks-Fischler-Shenker-Susskind (BFSS) and Ishibashi-Kawai-Kitazawa-Tsuchiya (IKKT) are supposed to provide a non-perturbative definition of $\mathrm{M}$ theory and type IIB string theory respectively [5, 20]. On the other hand, MMs are also useful laboratories for the study of structures which could be relevant from a low-energy point of view. Indeed, they generate a plethora of interesting solutions, corresponding to strings, D-branes and their interactions [5, 21], as well as to non-commutative/fuzzy spaces, such as fuzzy tori and spheres [22]. Such backgrounds naturally give rise to non-abelian gauge theories. Therefore it appears natural to pose the question whether it is possible to construct phenomenologically interesting particle physics models in this framework as well.

In particular, MMs also allow to explore ideas and lessons which initially arose in the context of orbifolds. An orbifold MM was proposed by Aoki-Iso-Suyama (AIS) in [23] as a particular projection of the IKKT model, and it is directly related to our construction in which fuzzy extra dimensions arise with trinification gauge theory [17]. By $\mathbb{Z}_{3}$-orbifolding, the original symmetry of the IKKT matrix model with matrix size $3 N \times 3 N$ is generally reduced from $S O(9,1) \times U(3 N)$ to $S O(3,1) \times U(N)^{3}$. This model is chiral and has $D=4, N=1$ supersymmetry of Yang-Mills type as well as an inhomogeneous supersymmetry specific to matrix models. The $\mathbb{Z}_{3}$-invariant fermion fields transform as bi-fundamental representations under the unbroken gauge symmetry exactly as in our constructions.

In the following we present first the field theoretic construction described above, which we then attempt to embed in the existing Orbifold MM [23] performing the appropriate modifications. We briefly speculate on the gravity that emerges out of this picture, and we leave for future work the phenomenological consequences resulting from such an embedding, noting that the fourdimensional non-commutativity appears as a new feature.

\section{Field theory orbifolds and fuzzy spheres}

Let us begin with a brief reminder of the application of orbifold techniques in field theory and their relation to the dynamical generation of fuzzy extra dimensions. The main motivation behind this concerns the possibility to obtain chiral matter when models for particle physics are engineered.

The starting point is a $S U(3 N) \mathscr{N}=4$ supersymmetric Yang-Mills (SYM) theory. This theory may be projected under the action of the discrete group $\mathbb{Z}_{3}$; this is essentially an orbifold projection. This procedure amounts to an embedding of the discrete symmetry into the $R$-symmetry of the original theory, which is $S U(4)_{R}$. It is well-known that depending on this embedding, projected theories with different amount of remnant supersymmetry may be obtained [24]. Indeed, a maximal 
embedding $\Gamma \in S U(4)_{R}$ breaks supersymmetry completely, while embeddings within an $S U(3)$ or $S U$ (2) part of the $R$-symmetry lead to $\mathscr{N}=1$ or $\mathscr{N}=2$ supersymmetric theories respectively. In the present work our attention is focused on the first of the latter two possibilities, which is in accord with our motivation of obtaining chiral theories.

A projection of the initial theory under the discrete symmetry $\mathbb{Z}_{3}$ corresponds to an $\mathscr{N}=1$ SYM theory where only the $\mathbb{Z}_{3}$-invariant fields survive. The details of this procedure in the present context may be found in [17]; here we just list the most important results. The original $\mathscr{N}=4 \mathrm{SYM}$ theory contains a vector superfield and three chiral superfields in $\mathscr{N}=1$ language. The component fields of the theory are the gauge fields $A_{\mu}, \mu=0, \ldots 3$ of the $S U(3 N)$ gauge group, three complex scalars $\phi^{i}, i=1,2,3$, trnasforming in the adjoint of the gauge group and in the vector of the global symmetry, and four Majorana fermions $\psi^{p}$, in the adjoint of the gauge group and the spinor of the global symmetry. After the projection, the resulting theory has a different gauge group and spectrum. Indeed, the gauge group is broken down to $H=S(U(N) \times U(N) \times U(N))$, while the surving scalar fields and fermions transform according to the representations

$$
3 \cdot((N, \bar{N}, 1)+(\bar{N}, 1, N)+(1, N, \bar{N}))
$$

under the non-abelian gauge group factors. This spectrum is evidently free of non-abelian gauge anomalies. Moreover, it is directly observed that the matter fields transform in chiral representations of the gauge group and they are replicated three times, thus leading to three chiral families.

Furthermore, the F-term scalar potential of the $\mathscr{N}=4$ SYM theory is

$$
V_{F}(\phi)=\frac{1}{4} \operatorname{Tr}\left(\left[\phi^{i}, \phi^{j}\right]^{\dagger}\left[\phi^{i}, \phi^{j}\right]\right),
$$

and it formally remains the same after the projection with the obvious difference that it describes only the interactions among the surviving fields. The D-term contribution to the scalar potential is given by $V_{D}=\frac{1}{2} D^{2}=\frac{1}{2} D^{I} D_{I}$, where the $\mathrm{D}$-terms have the form $D^{I}=\phi_{i}^{\dagger} T^{I} \phi^{i}$, with $T^{I}$ the generators of the representation of the corresponding chiral multiplets. The minimum of the full potential is obtained for vanishing F- and D-terms, which means that $\left[\phi^{i}, \phi^{j}\right]=0$. However, a very different class of vacua may be revealed upon adding a soft supersymmetry breaking sector in the theory. The scalar part of this sector is

$$
V_{S S B}=\frac{1}{2} \sum_{i} m_{i}^{2} \phi^{i^{\dagger}} \phi^{i}+\frac{1}{2} \sum_{i, j, k} h_{i j k} \phi^{i} \phi^{j} \phi^{k}+h . c .,
$$

and it respects the orbifold symmetry. After the addition of these soft terms the scalar potential of the theory becomes

$$
V=V_{F}+V_{S S B}+V_{D}
$$

which has the equivalent form

$$
V=\frac{1}{4}\left(F^{i j}\right)^{\dagger} F^{i j}+V_{D}
$$

for suitable parameters, where we have defined

$$
F^{i j}=\left[\phi^{i}, \phi^{j}\right]-i \varepsilon^{i j k}\left(\phi^{k}\right)^{\dagger}
$$


Since the first term is positive definite, the global minimum of the potential is obtained when the following relations hold,

$$
\begin{aligned}
{\left[\phi^{i}, \phi^{j}\right] } & =i \varepsilon_{i j k}\left(\phi^{k}\right)^{\dagger}, \\
{\left[\left(\phi^{i}\right)^{\dagger},\left(\phi^{j}\right)^{\dagger}\right] } & =i \varepsilon_{i j k} \phi^{k}, \\
\phi^{i}\left(\phi^{i}\right)^{\dagger} & =R^{2},
\end{aligned}
$$

where $\left(\phi^{i}\right)^{\dagger}$ denotes hermitean conjugation of the complex scalar field $\phi^{i}$ and $\left[R^{2}, \phi^{i}\right]=0$. The above relations are closely related to the usual fuzzy sphere. This can be seen by considering the untwisted fields $\tilde{\phi}_{i}$, defined by

$$
\phi^{i}=\Omega \tilde{\phi}^{i},
$$

for some $\Omega \neq 1$ which satisfies $\Omega^{3}=1,\left[\Omega, \phi^{i}\right]=0, \Omega^{\dagger}=\Omega^{-1}$ and $^{1}\left(\tilde{\phi}^{i}\right)^{\dagger}=\tilde{\phi}^{i}$, i.e. $\left(\phi^{i}\right)^{\dagger}=\Omega \phi^{i}$. Then (2.7) reduces to the ordinary fuzzy sphere relation

$$
\left[\tilde{\phi}^{i}, \tilde{\phi}^{j}\right]=i \varepsilon_{i j k} \tilde{\phi}^{k}
$$

generated by $\tilde{\phi}^{i}$, as well as to the relation $\tilde{\phi}^{i} \tilde{\phi}^{i}=R^{2}$.

Configurations of $\phi^{i}$ satisfying the relations (2.7) have the form

$$
\phi^{i}=\Omega\left(\mathbf{1}_{3} \otimes \lambda_{(N)}^{i}\right)
$$

where $\lambda_{(N)}^{i}$ denote the generators of $S U(2)$ in the $N$-dimensional irreducible representation and the matrix $\Omega$ is given by

$$
\Omega=U \otimes \mathbf{1}_{N}, \quad U=\left(\begin{array}{lll}
0 & 1 & 0 \\
0 & 0 & 1 \\
1 & 0 & 0
\end{array}\right), \quad U^{3}=\mathbf{1} .
$$

This solution completely breaks the gauge symmetry $S U(N)^{3}$ (one could say that it corresponds to the Higgs branch of the SYM theory). However, there exist solutions which do not break the gauge symmetry completely, such as

$$
\phi^{i}=\Omega\left(\mathbf{1}_{3} \otimes\left(\lambda_{(N-n)}^{i} \oplus 0_{n}\right)\right),
$$

where $0_{n}$ denotes the $n \times n$ matrix with vanishing entries. The gauge symmetry in this case is broken from $S U(N)^{3}$ down to $S U(n)^{3}$ and the vacuum is interpreted as $\mathbb{R}^{4} \times K_{F}$ with an internal fuzzy geometry $K_{F}$ of a twisted fuzzy sphere. Such vacua may lead to a phenomenologically interesting low-energy theory, as discussed in [17].

\section{Orbifold matrix models}

The IKKT matrix model was originally proposed in [5] as a non-perturbative definition of the type IIB superstring theory. It is a reduced matrix model defined by the following action functional,

$$
\mathscr{S}_{I K K T}=-\frac{1}{2 g^{2}} \operatorname{Tr}\left(\frac{1}{2}\left[X_{a}, X_{b}\right]\left[X^{a}, X^{b}\right]+\bar{\Psi} \Gamma^{a}\left[X_{a}, \Psi\right]\right),
$$

\footnotetext{
${ }^{1}$ This is understood before the orbifolding.
} 
where $X^{a}, a=0, \ldots, 9$ are ten hermitian matrices, $\Psi$ are 16-component Majorana-Weyl spinors and $g$ is a coupling parameter. Moreover, $\Gamma^{a}$ are 10-dimensional matrices furnishing the spinor representation of $S O(10)$, which is a symmetry of the Euclidean model inherited by its 10-dimensional origin. The full symmetry group of the above model contains the $U(N)$ gauge group (where actually $N \rightarrow \infty$ is understood) as well as the aforementioned $S O(10)$ global symmetry and a $\mathscr{N}=2$ supersymmetry. The action (3.1) may be varied with respect to the matrices $X_{a}$ and, setting $\Psi=0$, the resulting equations of motion are,

$$
\left[X_{b},\left[X^{a}, X^{b}\right]\right]=0 .
$$

Classical solutions of the model can be obtained in the case when the matrices are commuting, $\left[X^{a}, X^{b}\right]=0$, but also when they do not commute, $\left[X^{a}, X^{b}\right] \neq 0$; in the rest of this work we focus on the second possibility since the former one does not generate interesting dynamics. Furthermore, since we are going to describe non-commutative (NC) branes embedded in $\mathbb{R}^{10}$, let us consider a splitting of the ten matrices $X^{a}$ as

$$
X^{a}=\left(\begin{array}{c}
X^{\mu} \\
X^{i}
\end{array}\right), \quad \mu=0, \ldots, 2 d, \quad i=2 d+1, \ldots, 9 .
$$

Then the $X^{\mu}$ correspond to the world-volume directions on the branes and the $X^{i}$ correspond to the transverse displacements.

In the above notation, a solution of the equations (3.2) is given by

$$
X^{a}=\left(\begin{array}{c}
\bar{X}^{\mu} \\
0
\end{array}\right)
$$

where $\bar{X}^{\mu}$ are the generators of the Moyal-Weyl quantum plane $\mathbb{R}_{\theta}^{2 d}$, which satisfy the commutation relation

$$
\left[\bar{X}^{\mu}, \bar{X}^{v}\right]=i \theta^{\mu v}
$$

where $\theta^{\mu v}$ is a constant antisymmetric tensor. This solution preserves half of the supersymmetries of the IKKT model, and corresponds to a single non-commutative flat $(2 d-1)$-brane. Being a single brane, this solution is associated to an abelian gauge theory. Non-abelian gauge theories are implemented via direct generalization of the above solution,

$$
X^{a}=\left(\begin{array}{c}
\bar{X}^{\mu} \\
0
\end{array}\right) \otimes \mathbf{1}_{n}
$$

where $\mathbf{1}_{n}$ is the $n$-dimensional unit matrix. This configuration is interpreted as $n$ coincident branes carrying a non-abelian $U(n)$ gauge theory, similarly to ordinary D-branes [25].

The flat solutions $\mathbb{R}_{\theta}^{2 d}$ are special cases of NC branes with generic embedding in $\mathbb{R}^{10}$. Such generic branes are desribed by quantized embedding functions

$$
X^{a} \sim x^{a}: \quad \mathscr{M}^{2 n} \hookrightarrow \mathbb{R}^{10}
$$

of a $2 n$ dimensional submanifold. Furthermore,

$$
\left[X^{\mu}, X^{v}\right] \sim i\left\{x^{\mu}, x^{v}\right\}=i \theta^{\mu v}(x)
$$


is interpreted as a quantized Poisson structure on $\mathscr{M}^{2 n}$. Here $\sim$ denotes the semi-classical limit where commutators are replaced by Poisson brackets, and $x^{\mu}$ are locally independent coordinate functions chosen among the $x^{a}$. Thus we are considering quantized embedded Poisson manifolds $\left(\mathscr{M}^{2 n}, \theta^{\mu v}\right)$. The sub-manifold $\mathscr{M}^{2 n} \subset \mathbb{R}^{10}$ is equipped with a non-trivial induced metric

$$
g_{\mu v}(x)=\partial_{\mu} x^{a} \partial_{v} x^{b} \eta_{a b}
$$

via the pull-back of $\eta_{a b}$. The kinetic term for all (scalar, gauge and fermionic) fields in the MM on such a background $\mathscr{M}^{2 n}$ is governed (up to possible conformal factors) by the effective metric

$$
G^{\mu v} \sim \theta^{\mu \rho} \theta^{v \sigma} g_{\rho \sigma}
$$

so that $G_{\mu \nu}$ must be interpreted as gravitational metric. Since the embedding is dynamical, the model describes a dynamical theory of gravity, realized on dynamically determined submanifolds of $\mathbb{R}^{10}$.

The IKKT matrix model is very interesting in view of its conjectured merit to capture nonperturbative type IIB superstring theory. However, it is not obvious how one could relate it directly to the observed four-dimensional world and make contact with low-energy phenomenology. In particular, its main limitation is the difficulty in the description of chiral fermions, since it accommodates matter in real representations of the gauge group. The above problem may be addressed in a variety of ways. Here, motivated by the discussion of the previous section, we discuss the possibility to construct orbifold equivalents within the framework of matrix models. Such an approach was initially employed by Aoki, Iso and Suyama in [23]. Orbifold techniques in bottom-up stringy constructions, i.e. from a 4-dimensional perspective, were analyzed in [26] in the context of phenomenologically attractive model building with D-branes. Here we find it important to retain the 0-dimensional perspective of reduced matrix models and study orbifold techniques in this context.

In order to apply the aforementioned orbifold techniques in our case, we have to specify the embedding of some discrete symmetry in the full symmetry group $U(N) \times S O(10)$ of the IKKT model. The basic steps closely follow the corresponding discussion for field theories which was sketched in section 2. In the present context we discuss the discrete group $\mathbb{Z}_{3}$ too. Clearly there is a huge number of different embeddings which can be considered. However, in accordance with the lessons from string theory compactifications and field-theoretic orbifolds of $\mathscr{N}=4$ SYM theory $[27,24]$, it is reasonable to embed $\mathbb{Z}_{3}$ in the $S O(6)$ part of the global $S O(10)$ symmetry.

According to the above, let us choose six out of the nine space-like matrices of the IKKT model and write them in complex form $Z_{i}, i=1,2,3$,

$$
Z_{1}=X_{4}+i X_{5}, \quad Z_{2}=X_{6}+i X_{7}, \quad Z_{3}=X_{8}+i X_{9}
$$

Then the action of $\mathbb{Z}_{3}$ on the matrices is characterized by three integers $\left(a_{1}, a_{2}, a_{3}\right)$, one for each complex matrix [27]. Let us denote with

$$
\zeta \in \mathbb{Z}_{3}
$$

the abstract generator of $\mathbb{Z}_{3}$. The $\mathbb{Z}_{3}$ transformation is defined to act on the above complex matrices as

$$
\zeta\left(Z_{i}\right)=\omega^{a_{i}} Z_{i}
$$


On the other hand, its action on the rest four of the ten matrices is trivial, i.e.

$$
\zeta\left(X^{\mu}\right)=X^{\mu}
$$

The important point here is that the realization of the $\mathbb{Z}_{3}$ action on the indices, i.e. on $\mathbb{R}^{10}$, is chosen such that there is a 4-dimensional fixed subspace. This selects the spacetime brane, which is a solution of the IKKT model as we explained above. The brane solutions of the orbifolded model respect the $\mathbb{Z}_{3}$ symmetry as long as they do not move off the fixed plane. In order for a brane to be able to move off this plane, one has to add its image branes under the $\mathbb{Z}_{3}$ symmetry, which are two more branes. This extends the size of the matrices by three times and the gauge group at the origin appears initially to be $U(3 N)$.

Up to here we have only considered the $\mathbb{Z}_{3}$ action as far as the $S O(10)$ symmetry is concerned. However, the discrete group acts on the matrices with respect to the $U(3 N)$ gauge symmetry as well. Let us denote the action of $\mathbb{Z}_{3}$ on the $U(3 N)$ gauge sector as $\gamma \in U(3 N)$. This is necessarily an action through the adjoint representation, i.e. it is given by

$$
\begin{aligned}
\gamma\left(X^{\mu}\right) & =\gamma X^{\mu} \gamma^{-1} \\
\gamma\left(Z_{i}\right) & =\gamma Z_{i} \gamma^{-1}
\end{aligned}
$$

A convenient representation for $\gamma$ is

$$
\gamma=\left(\begin{array}{lll}
\mathbf{1}_{N} & 0 & 0 \\
0 & \omega \mathbf{1}_{N} & 0 \\
0 & 0 & \omega^{2} \mathbf{1}_{N}
\end{array}\right) \equiv V \otimes \mathbf{1}_{N},
$$

where we have defined the $3 \times 3$ matrix

$$
V=\left(\begin{array}{lll}
1 & 0 & 0 \\
0 & \omega & 0 \\
0 & 0 & \omega^{2}
\end{array}\right)
$$

which satisfies $V^{3}=\mathbf{1}_{3}$.

In the same spirit, the action of $\mathbb{Z}_{3}$ on the spinor matrices may be defined. In particular, the corresponding actions are

$$
\zeta(\Psi)=\omega^{b_{i}} \Psi
$$

for the $S O(6)$ symmetry and

$$
\gamma(\Psi)=\gamma \Psi \gamma^{-1}
$$

for the gauge summetry. In eq. (3.16) the parameters $b$ are four integers, one for each of the 4-spinors, which are specifically related to the $a_{i}$ as

$$
b_{0}=\sum a_{i}, \quad b_{i}=a_{i}-a_{i+1}-a_{i+2} .
$$

The orbifold matrix model is defined as the invariant sector of the IKKT model under the full action of $\mathbb{Z}_{3}$ [23]. In order to be more specific, let us consider the matrices $Z_{i}$ and write them in a $3 \times 3$ block form,

$$
Z_{i}=\left(\begin{array}{ccc}
z_{i}^{(11)} & z_{i}^{(12)} & z_{i}^{(13)} \\
z_{i}^{(21)} & z_{i}^{(22)} & z_{i}^{(23)} \\
z_{i}^{(31)} & z_{i}^{(32)} & z_{i}^{(33)}
\end{array}\right)
$$


Similar expressions will be considered for the matrices $X^{\mu}$ and $\Psi$ with entries $x^{\mu(i j)}$ and $\psi^{(i j)}$ respectively. The embedding of $\mathbb{Z}_{3}$ in $S O(10)$ that we consider here translates into the following action on the $Z_{i}$ matrices,

$$
Z_{i}^{\prime}=\omega^{a_{i}} \gamma Z_{i} \gamma^{-1}
$$

Invariance under the $\mathbb{Z}_{3}$ action, which is described by the condition $Z_{i}^{\prime}=Z_{i}$, then implies that the following matrix equation is satisfied,

$$
\left(\begin{array}{lll}
z_{i}^{(11)} & \omega z_{i}^{(12)} & \omega^{2} z_{i}^{(13)} \\
z_{i}^{(21)} & \omega z_{i}^{(22)} & \omega^{2} z_{i}^{(23)} \\
z_{i}^{(31)} & \omega z_{i}^{(32)} & \omega^{2} z_{i}^{(33)}
\end{array}\right)=\omega^{a_{i}}\left(\begin{array}{lll}
z_{i}^{(11)} & z_{i}^{(12)} & z_{i}^{(13)} \\
\omega z_{i}^{(21)} & \omega z_{i}^{(22)} & \omega z_{i}^{(23)} \\
\omega^{2} z_{i}^{(31)} & \omega^{2} z_{i}^{(32)} & \omega^{2} z_{i}^{(33)}
\end{array}\right) .
$$

Clearly the embedding depends crucially on the integers $a_{i}$. We shall consider the following two cases:

1. $a_{i}=(1,1,1) \bmod 3$.

In this case the equation (3.20) becomes,

$$
\left(\begin{array}{lll}
z_{i}^{(11)} & \omega z_{i}^{(12)} & \omega^{2} z_{i}^{(13)} \\
z_{i}^{(21)} & \omega z_{i}^{(22)} & \omega^{2} z_{i}^{(23)} \\
z_{i}^{(31)} & \omega z_{i}^{(32)} & \omega^{2} z_{i}^{(33)}
\end{array}\right)=\left(\begin{array}{lll}
\omega z_{i}^{(11)} & \omega z_{i}^{(12)} & \omega z_{i}^{(13)} \\
\omega^{2} z_{i}^{(21)} & \omega^{2} z_{i}^{(22)} & \omega^{2} z_{i}^{(23)} \\
z_{i}^{(31)} & z_{i}^{(32)} & z_{i}^{(33)}
\end{array}\right)
$$

which means that only three entries of $Z_{i}$ remain non-zero and in particular

$$
Z_{i}=\left(\begin{array}{lll}
0 & z_{i}^{(12)} & 0 \\
0 & 0 & z_{i}^{(23)} \\
z_{i}^{(31)} & 0 & 0
\end{array}\right)
$$

Moreover, as far as the matrices $X^{\mu}$ are concerned, the invariance condition is

$$
X^{\prime \mu}=\gamma X^{\mu} \gamma^{-1}
$$

and therefore we obtain

$$
X^{\mu}=\left(\begin{array}{lll}
x^{\mu(11)} & 0 & 0 \\
0 & x^{\mu(22)} & 0 \\
0 & 0 & x^{\mu(33)}
\end{array}\right) .
$$

Similar results apply for the fermions. The integers $b_{i}$ turn out to be $b_{0}=0 \bmod 3$ and $b_{i}=(1,1,1) \bmod 3$ and therefore

$$
\Psi_{0}=\left(\begin{array}{lll}
\psi_{0}^{(11)} & 0 & 0 \\
0 & \psi_{0}^{(22)} & 0 \\
0 & 0 & \psi_{0}^{(33)}
\end{array}\right), \quad \Psi_{i}=\left(\begin{array}{lll}
0 & \psi_{i}^{(12)} & 0 \\
0 & 0 & \psi_{i}^{(23)} \\
\psi_{i}^{(31)} & 0 & 0
\end{array}\right) .
$$

According to the above analysis, there are some immediate results concerning the gauge group and the spectrum of the projected theory, as well as its amount of supersymmetry. Indeed, according to eq. (3.24) the gauge group of the resulting theory is $U(N)^{3}$. Moreover, 
according to eqs. (3.22) and (3.25) the bosonic and the fermionic matrices $Z_{i}$ and $\Psi_{i}$ of the theory transform under the gauge group in the following bifundamental representations,

$$
3 \cdot((N, \bar{N}, 1)+(\bar{N}, 1, N)+(1, N, \bar{N})) .
$$

As far as supersymmetry is concerned, there is only one gaugino left in the projected theory, the $\Psi_{0}$, and therefore the theory is $\mathscr{N}=1$ supersymmetric. The identical spectrum for the bosonic and fermionic matrices in eq. (3.26) just confirms this fact. Therefore, the spectrum contains one vector supermultiplet and three chiral supermultiplets.

2. $a_{i}=(1,2,0) \bmod 3$.

In this case the equation (3.20) has to be split in three equations, one for each $i$. The result is that the three matrices $Z_{i}$ assume the following form,

$$
Z_{1}=\left(\begin{array}{ccc}
0 & z_{1}^{(12)} & 0 \\
0 & 0 & z_{1}^{(23)} \\
z_{1}^{(31)} & 0 & 0
\end{array}\right), Z_{2}=\left(\begin{array}{ccc}
0 & 0 & z_{2}^{(13)} \\
z_{2}^{(21)} & 0 & 0 \\
0 & z_{2}^{(32)} & 0
\end{array}\right), Z_{3}=\left(\begin{array}{ccc}
z_{3}^{(11)} & 0 & 0 \\
0 & z_{3}^{(22)} & 0 \\
0 & 0 & z_{3}^{(33)}
\end{array}\right)
$$

Moreover, while for the matrices $X^{\mu}$ the previous result remains unaltered, for the fermionic matrices we obtain the integers $b_{0}=0 \bmod 3$ and $b_{i}=(2,1,0) \bmod 3$. This gives the result

$$
\begin{aligned}
& \Psi_{0}=\left(\begin{array}{lll}
\psi_{0}^{(11)} & 0 & 0 \\
0 & \psi_{0}^{(22)} & 0 \\
0 & 0 & \psi_{0}^{(33)}
\end{array}\right), \quad \Psi_{3}=\left(\begin{array}{lll}
\psi_{3}^{(11)} & 0 & 0 \\
0 & \psi_{3}^{(22)} & 0 \\
0 & 0 & \psi_{3}^{(33)}
\end{array}\right) \\
& \Psi_{1}=\left(\begin{array}{ccc}
0 & \psi_{1}^{(12)} & 0 \\
0 & 0 & \psi_{1}^{(23)} \\
\psi_{1}^{(31)} & 0 & 0
\end{array}\right), \quad \Psi_{2}=\left(\begin{array}{lll}
0 & \psi_{2}^{(12)} & 0 \\
0 & 0 & \psi_{2}^{(23)} \\
\psi_{2}^{(31)} & 0 & 0
\end{array}\right) .
\end{aligned}
$$

Presently we end up with $\mathscr{N}=2$ supersymmetry, which is reminiscent of a configuration $\mathbb{R}^{6} \times \mathbb{C}^{2} / \mathbb{Z}_{2}$. The spectrum consists of a $\mathscr{N}=2$ vector supermultiplet and a $\mathscr{N}=2$ hypermultiplet.

Out of the above two possibilities, we shall focus on the first one. The reason is that the second case leads to a $\mathscr{N}=2$ supersymmetric gauge theory on the space-time brane and therefore it cannot be phenomenologically viable due to the absence of chiral matter. On the other hand, the first case corresponds to a $\mathscr{N}=1$ gauge theory with chiral matter content and therefore it has a promising spectrum.

One illustrating way to rewrite the above matrices is provided by the shift and clock matrices

$$
U=\left(\begin{array}{lll}
0 & 1 & 0 \\
0 & 0 & 1 \\
1 & 0 & 0
\end{array}\right), \quad V=\left(\begin{array}{lll}
1 & 0 & 0 \\
0 & \omega & 0 \\
0 & 0 & \omega^{2}
\end{array}\right)
$$

These matrices satisfy the relations

$$
\begin{aligned}
U U^{\dagger} & =1, \quad \text { i.e. } \quad U^{\dagger}=U^{-1}, \\
U^{2} & =U^{-1},
\end{aligned}
$$


and similarly for $V$. Moreover, they satisfy

$$
U V=\omega V U
$$

Having defined these auxilliary matrices, it is straightforward to see that one can write the configurations compatible with the orbifold constraint in the following way

$$
\begin{aligned}
X_{\mu} & =x_{\mu}^{I} \otimes V^{I} \\
Z_{i} & =z_{i}^{I} \otimes V^{I} U .
\end{aligned}
$$

These representations of the bosonic matrices are useful in order to determine the gauge group and the spectrum of the theory, as it was exhibited in the previous section. However, the interpretation of the orbifold states becomes more illuminating in a different basis where

$$
\tilde{\gamma}=\left(\begin{array}{lll}
0 & \mathbf{1}_{N} & 0 \\
0 & 0 & \mathbf{1}_{N} \\
\mathbf{1}_{N} & 0 & 0
\end{array}\right)=\mathbf{1}_{N} \otimes U .
$$

This just corresponds to an alternative choice for the representative element of the action of $\mathbb{Z}_{3}$ on the matrices. Indeed, it cubes to the unit matrix and its eigenvalues are $\left(1, \omega, \omega^{2}\right)$.

The new choice of representative element of $\mathbb{Z}_{3}$ is of course physically equivalent, but leads to different forms for the relevant matrices. Indeed, the new invariance conditions

$$
\begin{aligned}
X^{\mu} & =\tilde{\gamma} X^{\mu} \tilde{\gamma}^{-1}, \\
Z_{i} & =\omega^{a_{i}} \tilde{\gamma} Z_{i} \tilde{\gamma}^{-1},
\end{aligned}
$$

are now solved by matrices of the general form

$$
\begin{aligned}
X^{\mu} & =\left(\begin{array}{lll}
x^{\mu(11)} & x^{\mu(12)} & x^{\mu(13)} \\
x^{\mu(13)} & x^{\mu(11)} & x^{\mu(12)} \\
x^{\mu(12)} & x^{\mu(13)} & x^{\mu(11)}
\end{array}\right), \\
Z_{i} & =\left(\begin{array}{lll}
z_{i}^{(11)} & z_{i}^{(12)} & z_{i}^{(13)} \\
\omega^{2} z_{i}^{(13)} & \omega^{2} z_{i}^{(11)} & \omega^{2} z_{i}^{(12)} \\
\omega z_{i}^{(12)} & \omega z_{i}^{(13)} & \omega z_{i}^{(11)}
\end{array}\right) .
\end{aligned}
$$

Clearly the gauge group and the spectrum are more obscure in this picture. Now, let us write these matrices in a form analogous to (3.34) and (3.35). For $X^{\mu}$ we have

$$
X^{\mu}=x^{\mu(11)} \otimes 1+x^{\mu(12)} \otimes U+x^{\mu(13)} \otimes U^{2} \equiv x^{\mu I} \otimes U^{I} .
$$

Similarly, for $Z_{i}$ we obtain

$$
Z_{i}=z_{i}^{(11)} \otimes V^{2}+z_{i}^{(12)} \otimes V^{2} U+z_{i}^{(13)} \otimes V^{2} U^{2} \equiv z_{i}^{I} \otimes V^{2} U^{I},
$$

where

$$
V^{2} U=\left(\begin{array}{lll}
0 & 1 & 0 \\
0 & 0 & \omega^{2} \\
\omega & 0 & 0
\end{array}\right), \quad V^{2} U^{2}=\left(\begin{array}{lll}
0 & 0 & 1 \\
\omega^{2} & 0 & 0 \\
0 & \omega & 0
\end{array}\right)
$$


Let us return now to the action and the equations of motion of the matrix model and discuss their form after the orbifold projection. The action of the IKKT model is given by eq. (3.1) and its equations of motion by eq. (3.2). It is not surprising that the corresponding action and equations of motion for the orbifold matrix model are typically given by the same equations, albeit restricted to the $\mathbb{Z}_{3}$-invariant sector. However, it is very instructive to rewrite the action in a more illustrative form, which takes into account the fact that the orbifold group acts differently on $X^{\mu}$ and $Z_{i}$.

The bosonic part of the action (3.1) can be brought in the following form,

$$
\begin{aligned}
S_{B}=-\frac{1}{4 g^{2}} \operatorname{Tr}( & {\left[X_{\mu}, X_{V}\right]\left[X^{\mu}, X^{v}\right]+2\left[X_{\mu}, Z_{i}\right]\left[X^{\mu}, Z_{i}^{\dagger}\right] } \\
& \left.+\frac{1}{2}\left[Z_{i}, Z_{i}^{\dagger}\right]\left[Z_{j}, Z_{j}^{\dagger}\right]+2\left[Z_{i}^{\dagger}, Z_{j}^{\dagger}\right]\left[Z_{i}, Z_{j}\right]\right) .
\end{aligned}
$$

The corresponding equations of motion are the following,

$$
\begin{gathered}
{\left[X_{\mu},\left[X_{\mu}, X_{V}\right]\right]+\frac{1}{2}\left(\left[Z_{i},\left[Z_{i}^{\dagger}, X_{V}\right]\right]+\left[Z_{i}^{\dagger},\left[Z_{i}, X_{V}\right]\right]\right)=0} \\
{\left[X_{\mu},\left[X_{\mu}, Z_{j}\right]\right]+\frac{1}{2}\left(\left[Z_{i},\left[Z_{i}^{\dagger}, Z_{j}\right]\right]+\left[Z_{i}^{\dagger},\left[Z_{i}, Z_{j}\right]\right]\right)=0 .}
\end{gathered}
$$

Let us now discuss the solutions of these equations of motion. The simplest solutions correspond to commuting matrices $\left[X^{a}, X^{b}\right]=0$, exactly as in the IKKT model. which we do not discuss here. Instead, we shall proceed to the analysis of non-commutative solutions. There are two types of non-commutative solutions of the orbifold matrix model, which we shall describe in the basis given by eq. (3.36).

Higgs branch. The first class of solutions is given by the following background ${ }^{2}$,

$$
\begin{aligned}
X^{\mu} & =\left(x^{\mu} \otimes \mathbf{1}_{n}\right) \otimes \mathbf{1}_{3}, \\
Z_{i} & =\left(z_{i} \otimes \mathbf{1}_{n}\right) \otimes V^{2},
\end{aligned}
$$

where the commutation relations of the matrices are given by

$$
\begin{aligned}
{\left[x^{\mu}, x^{v}\right] } & =i \theta^{\mu v}, \\
{\left[x^{\mu}, z_{i}\right] } & =0 \\
{\left[z_{i}, z_{j}^{\dagger}\right] } & =i \theta_{i j} \\
{\left[z_{i}, z_{j}\right] } & =\left[z_{i}^{\dagger}, z_{j}^{\dagger}\right]=0 .
\end{aligned}
$$

This vacuum corresponds to the $I=0$ sector of the eqs. (3.41) and (3.42) and in the (noncommutative) super-Yang-Mills language it is the Higgs branch of the theory. The fluctuations around this vacuum lead to the corresponding effective action [23]. Since the internal bosonic matrices $Z_{i}$ acquire a vev, the original gauge symmetry $U(N)^{3}$ is spontaneously broken. In fact, the $U(N)$ IKKT model is recovered for large $z_{i}[23]$. Therefore the theory is not chiral in the Higgs branch. On the other hand, it admits brane embeddings with general geometry, which is strongly suggestive for gravity.

\footnotetext{
${ }^{2}$ The parentheses are of course not necessary but they aid in the better comprehension of the solution.
} 
Coulomb branch. A second solution is given by the background

$$
\begin{aligned}
X^{\mu} & =\left(x^{\mu I} \otimes \mathbf{1}_{n}\right) \otimes U^{I}, \\
Z_{i} & =0,
\end{aligned}
$$

with the only non-trivial commutation relation being identical to (3.48). This class of solutions corresponds to the Coulomb branch of the corresponding super-Yang-Mills theory and it is restricted on the orbifold fixed point. In the Coulomb branch the vevs of the $Z_{i}$ are zero and therefore the gauge symmetry $U(N)^{3}$ remains intact, i.e. the chiral theory is retained. However, since the branes are restricted to the orbifold fixed plane, gravity appears to be decoupled. An obvious challenge is to reconcile these seemingly incompatible pictures of chiral gauge theory and gravity, which will be addressed further in section 4.1.

\section{Modifications and fuzzy extra dimensions}

Here we would like to reveal and study another class of solutions, which are obtained after a particular modification of the above model. These solutions will correspond to fuzzy spheres, i.e. they will involve Lie-type non-commutativity. It is well-known that such solutions can be obtained by adding some appropriate terms in the matrix model action [22] corresponding to type IIB fluxes as discussed by Myers [28]. Such terms do not spoil the covergence of the related matrix integrals and therefore the model remains in the same topological class as shown in [29]. Moreover, such a modification is reminiscent of the addition of the soft supersymmetry breaking sector in the field theoretical context as we discussed in section 2.

In the present case, the terms which will be added in order to modify the matrix model are the following,

$$
V_{f l u x}=-2 \alpha^{2} \operatorname{Tr}\left(Z_{i}^{\dagger} Z_{i}\right)-i \alpha \varepsilon_{i j k} \operatorname{Tr}\left(\left[Z_{i}, Z_{j}\right] Z_{k}\right)+\text { h.c. }
$$

Then, if

$$
V=\frac{1}{2} \operatorname{Tr}\left(\left[Z_{i}, Z_{i}^{\dagger}\right]\left[Z_{j}, Z_{j}^{\dagger}\right]\right)+2 \operatorname{Tr}\left(\left[Z_{i}^{\dagger}, Z_{j}^{\dagger}\right]\left[Z_{i}, Z_{j}\right]\right),
$$

it is straightforward to show that the full part of the action involving the internal bosons takes the following form,

$$
V_{f u l l}=V+V_{f l u x}=\operatorname{Tr}\left(F_{i j}^{\dagger} F_{i j}\right)+D^{2},
$$

where

$$
F_{i j}=\left[Z_{i}, Z_{j}\right]-i \alpha \varepsilon_{i j k} Z_{k}^{\dagger}
$$

and

$$
D=\left[Z_{i}, Z_{i}^{\dagger}\right]
$$

Then the minimum of the action is obtained when

$$
\left\langle F_{i j}\right\rangle=0 \quad \text { and } \quad\langle D\rangle=0,
$$


i.e. when the following algebra is satisfied by the vacuum values of the $Z_{i}$ and the $Z_{i}^{\dagger}$,

$$
\begin{aligned}
{\left[Z_{i}, Z_{j}\right] } & =i \alpha \varepsilon_{i j k} Z_{k}^{\dagger}, \\
{\left[Z_{i}^{\dagger}, Z_{j}^{\dagger}\right] } & =i \alpha \varepsilon_{i j k} Z_{k}, \\
{\left[Z_{i}, Z_{i}^{\dagger}\right] } & =0,
\end{aligned}
$$

In order to determine the solutions of the matrix model under consideration, let us write down the $Z_{i}$ in the following suggestive form (no summation in the index $i$ ),

$$
Z_{i}=Y_{i} \otimes f_{i}(U, V),
$$

with $U$ and $V$ as before. Each $f_{i}(U, V)$ is a matrix-valued function of the matrices $U$ and $V$ and can be interpreted as a scalar function on an auxilliary fuzzy torus described by these two matrices. We assume that $Y_{i}$ are hermitian matrices, i.e.

$$
Y_{i}^{\dagger}=Y_{i}
$$

Moreover, due to the $\mathbb{Z}_{3}$ projection and the resulting form of the $Z_{i}$, the functions $f_{i}(U, V)$ must have the following special form,

$$
f_{i}(U, V)=a_{i} U+b_{i} U V+c_{i} U V^{2},
$$

where $a_{i}, b_{i}, c_{i}$ are complex coefficients. Therefore, the matrices and their conjugate ones have the form,

$$
\begin{aligned}
Z_{i} & =Y_{i} \otimes\left(a_{i} U+b_{i} U V+c_{i} U V^{2}\right), \\
Z_{i}^{\dagger} & =Y_{i} \otimes\left(a_{i}^{*} U^{2}+b_{i}^{*} V^{2} U^{2}+c_{i}^{*} V U^{2}\right) .
\end{aligned}
$$

Clearly, due to (4.7) and (4.8) the complex coefficients should satisfy certain constraints. Here we shall not go further into that since it does not add to the discussion. We shall therefore just consider the simplest and most obvious solution to the forementioned constraints, which is

$$
a_{i}=1, \quad b_{i}=c_{i}=0 \quad \text { for every } \quad i=1,2,3 .
$$

Furthermore, according to the commutation relations between the $Z_{i}$ in the vacuum, it is also true that

$$
\begin{aligned}
{\left[Y_{i}, Y_{j}\right] } & =i \alpha \varepsilon_{i j k} Y_{k}, \\
Y_{i} Y_{i} & =R^{2} .
\end{aligned}
$$

Let us now turn back to the full matrix model and combine the basic brane solution we described with the twisted $S_{F}^{2}$ solution for the internal bosonic degrees of freedom. The full bosonic sector of the matrix model, which combines the external and the internal matrices considered above, may be written as

$$
\begin{aligned}
S_{B}=-\frac{1}{4 g^{2}} \operatorname{Tr}( & {\left[X_{\mu}, X_{v}\right]\left[X^{\mu}, X^{v}\right]+2\left[X_{\mu}, Z_{i}\right]\left[X^{\mu}, Z_{i}^{\dagger}\right] } \\
& +\frac{1}{2}\left[Z_{i}, Z_{i}^{\dagger}\right]\left[Z_{j}, Z_{j}^{\dagger}\right]+2\left[Z_{i}^{\dagger}, Z_{j}^{\dagger}\right]\left[Z_{i}, Z_{j}\right] \\
& \left.-2 \alpha^{2} Z_{i}^{\dagger} Z_{i}-i \alpha \varepsilon_{i j k}\left[Z_{i}, Z_{j}\right] Z_{k}+\text { h.c. }\right) .
\end{aligned}
$$


It is now straightforward to combine the solutions we found previously into the following solution for the full model:

$$
\begin{aligned}
{\left[X_{\mu}, X_{v}\right] } & =i \theta_{\mu v} \\
{\left[Z_{i}, Z_{j}\right] } & =i \alpha \varepsilon_{i j k} Z_{k}^{\dagger}, \\
{\left[Z_{i}^{\dagger}, Z_{j}^{\dagger}\right] } & =i \alpha \varepsilon_{i j k} Z_{k}, \\
{\left[Z_{i}, Z_{i}^{\dagger}\right] } & =0, \\
{\left[X_{\mu}, Z_{i}\right] } & =0 \\
{\left[X_{\mu}, Z_{i}^{\dagger}\right] } & =0 .
\end{aligned}
$$

We can immediately write down the vacuum solutions for the different matrices,

$$
\begin{aligned}
X_{\mu} & =Y_{\mu} \otimes \mathbf{1}, \\
Z_{i} & =Y_{i} \otimes U, \\
Z_{i}^{\dagger} & =Y_{i} \otimes U^{2} .
\end{aligned}
$$

These are completely analogous to the solutions (2.12) of the chiral gauge theory considered in section 2. In particular, they may lead to an extension of the MSSM as discussed in [17], and therefore represent phenomenologically interesting solutions of the orbifold matrix model.

Let us now consider perturbations around the above vacuum solution. This task will determine the effective action on the resulting geometry. The relevant expansions are the following,

$$
\begin{aligned}
\delta X_{\mu} & =A_{\mu}^{I} \otimes V^{I}, \\
\delta Z_{i} & =\phi_{i}^{I} \otimes U V^{I}, \\
\delta Z_{i}^{\dagger} & =\phi_{i}^{\dagger I} \otimes V^{-I} U^{2} .
\end{aligned}
$$

where summation is implied for the index $I$. Note that for the fields $\left(A_{\mu}, \phi_{i}, \phi_{i}^{\dagger}\right) I$ is a labelling index, while for the matrices $U, V$ it is a power.

Let us now insert these expansions in the action (3.44). After some algebra and some cancellations we obtain the following result for the quadratic terms in the fields,

$$
\begin{aligned}
S_{B}^{(2)}=-\frac{3}{4 g^{2}} \operatorname{Tr}\{ & \left(\left[Y_{\mu}, A_{v}^{I}\right]-\left[Y_{v}, A_{\mu}^{-I}\right]\right)^{2}+2 i \theta_{\mu v} A_{\mu}^{I} A_{v}^{-I} \\
& +2\left(\omega^{I}+\omega^{-I}\right) Y_{i} A_{\mu}^{I} Y_{i} A_{\mu}^{-I}-2 Y_{i}^{2} A_{\mu}^{I} A_{\mu}^{-I} \\
& +2\left[Y_{\mu}, \phi_{i}^{I}\right] A_{\mu}^{-I} Y_{i}-2 \omega^{I}\left[Y_{\mu}, \phi_{i}^{I}\right] Y_{i} A_{\mu}^{-I} \\
& +2 \omega^{-I}\left[Y_{\mu}, \phi_{i}^{\dagger I}\right] A_{\mu}^{I} Y_{i}-2\left[Y_{\mu}, \phi_{i}^{\dagger I}\right] Y_{i} A_{\mu}^{I} \\
& -2 \alpha^{2} \phi_{i}^{\dagger I} \phi_{i}^{I}+2\left[Y_{\mu}, \phi_{i}^{\dagger I}\right]\left[Y_{\mu}, \phi_{i}^{I}\right] \\
& +Y_{i} Y_{j}\left(\phi_{j}^{\dagger I} \phi_{i}^{I}+\phi_{j}^{I} \phi_{i}^{\dagger I}+4 \phi_{i}^{\dagger I} \phi_{j}^{I}+4 \phi_{i}^{I} \phi_{j}^{\dagger I}\right)-4 Y_{i}^{2}\left(\phi_{j}^{I} \phi_{j}^{\dagger I}+\phi_{j}^{\dagger I} \phi_{j}^{I}\right) \\
& -\omega^{-I} Y_{i} \phi_{i}^{\dagger I} Y_{j} \phi_{j}^{J}-\omega^{I} Y_{i} \phi_{j}^{I} Y_{j} \phi_{i}^{\dagger I} \\
& -4 \omega^{I} Y_{i} \phi_{j}^{\dagger I} Y_{j} \phi_{i}^{I}-4 \omega^{-I} Y_{i} \phi_{i}^{I} Y_{j} \phi_{j}^{\dagger I}+4\left(\omega^{I}+\omega^{-I}\right) Y_{i} \phi_{j}^{\dagger I} Y_{i} \phi_{j}^{I} \\
& +\omega^{-I}\left(2 i \alpha \varepsilon_{i j k} Y_{k}-Y_{i} Y_{j}\right) \phi_{j}^{I} \phi_{i}^{-I} \\
& \left.+\frac{1}{2} Y_{i} \phi_{j}^{-I} Y_{j} \phi_{i}^{I}+\frac{1}{2} Y_{i} \phi_{i}^{I} Y_{j} \phi_{j}^{-I}+\text { h.c. }\right\} .
\end{aligned}
$$




\subsection{Relation with gravity and discussion}

As explained in section 3, the effective metric on brane solutions $\mathscr{M} \subset \mathbb{R}^{10}$ of the matrix model is governed by the metric $G^{\mu v}$ (3.7), which involves contributions from the brane embedding, as well as from the Poisson structure. In the orbifold matrix model, we focused primarily on branes $M^{4} \times K \subset \mathbb{R}^{10}$, where the embedding of the 4-dimensional brane is restricted to the orbifold fixed plane $\mathbb{R}^{4} \subset \mathbb{R}^{10}$. This restriction may be relaxed in the Higgs branch upon introducing mirror images under $\mathbb{Z}_{3}$, however then chirality is lost. Therefore the embedding degrees of freedom are constrained on a chiral background, which appears to pose a problem for gravity.

To shed new light on this issue, we note that the recovery of the IKKT model from the orbifold AIS model in the Higgs branch as discussed in [23] is very similar to an interesting observation by Ibanez [30]. In that paper, it was pointed out that a certain $\mathbb{Z}_{3}$ orientifold model obtained from IIB string theory gives rise to a $N=1, S U(N)^{3}$ chiral theory, which is continuously connected to a model with $N=4$ global supersymmetry by giving a vev to all the fields. Then the $S U(N)^{3}$ is broken to the diagonal $S U(N)$. The spectrum includes 3 adjoint chiral superfields under $S U(N)$ with the adequate Yukawa couplings, such that the massless $S U(N)$ charged spectrum has global $N=4$ supersymmetry (as well as a number of $S U(N) \operatorname{singlet}$ chiral $N=1$ supermultiplets that do not fall into $N=4$ supermultiplets). Therefore in a sense the $S U(N)^{3}$ charged sector of the theory has $N=4$ global supersymmetry and it is not chiral, however it bocomes chiral and only $N=1$ supersymmetric in some points of the moduli space. For momenta smaller than the size of the vevs one has a subsector of the theory with $N=4$ supersymmetry, and for momenta higher than those of the vevs the theory loses this extended supersymmetry and has only $N=1$. This is completely analogous to what happens in the orbifold matrix model, which is non-chiral in the Higgs branch (hence at low energies) and emergent gravity is expected to arise, while the chiral $N=1$ theory is recovered at a special point in the moduli space.

There may also be a different way to recover gravity even for the chiral vacua with the brane sitting on the orbifold fixed point, following the mechanism proposed in [31]. It turns out that for compactifications of the type $M^{4} \times K \subset \mathbb{R}^{10}$ with $\left[X^{\mu}, Y^{i}\right] \neq 0$, a form of 4-dimensional gravity can arises from the moduli degrees of freedom of $K$, which are transmitted to the non-compact spacetime $M^{4}$ via the Poisson structure $\left[X^{\mu}, Y^{i}\right] \sim i \theta^{\mu i} \neq 0$. Such a non-vanishing mixing between spacetime and internal coordinates is naturally obtained by giving the internal dimensions non-vanishing angular momentum, rotating along space-time. In the orbifold case, such spacetime-dependent twisted fuzzy extra dimensions can be obtained by modifying (4.20) as follows

$$
Z_{i}=e^{i k_{\mu} X^{\mu}} Y^{i} \otimes U
$$

where $Y^{i}$ is a hermitian generator of a fuzzy sphere. This is clearly compatible with the orbifold constraints, and satisfies the algebra

$$
\left[Z_{i}, Z_{j}\right]=i \alpha(X) \varepsilon_{i j k} Z_{k}^{\dagger}, \quad \alpha(X)=\alpha_{0} e^{i k_{\mu} X^{\mu}} .
$$

Note that in the case of Minkowski signature, these can be solutions of the orbifold matrix model for suitable time-like $k_{\mu}$, even without adding cubic or quadratic potential in the matrix model. More generally, one could take any solution of type $M^{4} \times K$ of the IKKT mode, and obtain a solution of the orbifold model by adding mirror images under $\mathbb{Z}^{3}$. The moduli of the compactification translate 
into a gravitational degrees of freedom via $\theta^{\mu i}$, contributing to gravity on the brane; we refer to [31] for a more detailed discussion. Although for the present simple compactification the resulting gravity may not be realistic, it might become realistic for more sophisticated compactifications. The main point is that there exists a mechanism for brane gravity within Yang-Mills matrix models which does carry over to the case of orbifold matrix model, however a more detailed investigation is required.

Acknowledgments. This work was partially supported by the SFB-Transregio TR33 "The Dark Universe" (Deutsche Forschungsgemeinschaft), the European Union 7th network program "Unification in the LHC era" (PITN-GA-2009-237920) and by the NTUA's programme supporting basic research PEVE 2009 and 2010. The work of HS was supported in part by the Austrian Science Fund (FWF) under the contract P21610-N16, and in part by a CCNY/Lehman CUNY collaborative grant.

\section{References}

[1] M. B. Green, J. H. Schwarz and E. Witten, Cambridge, Uk: Univ. Pr. ( 1987) 469 P. ( Cambridge Monographs On Mathematical Physics);

D. Lust and S. Theisen, Lect. Notes Phys. 346 (1989) 1;

J. Polchinski, Cambridge, UK: Univ. Pr. (1998) 531 p;

K. Becker, M. Becker and J. H. Schwarz, Cambridge, UK: Cambridge Univ. Pr. (2007) 739 p;

E. Kiritsis, Princeton University Press, 2007.

[2] A. Connes, Academic Press 1994;

J. Madore, Lond. Math. Soc. Lect. Note Ser 257, 1 (2000).

[3] A. Connes, M. R. Douglas and A. S. Schwarz, JHEP 9802 (1998) 003 [hep-th/9711162].

[4] N. Seiberg and E. Witten, JHEP 9909 (1999) 032 [hep-th/9908142].

[5] N. Ishibashi, H. Kawai, Y. Kitazawa and A. Tsuchiya, Nucl. Phys. B 498 (1997) 467 [arXiv:hep-th/9612115].

[6] T. Filk, Phys. Lett. B 376 (1996) 53;

J. C. Varilly and J. M. Gracia-Bondia, Int. J. Mod. Phys. A 14 (1999) 1305 [hep-th/9804001]; M. Chaichian, A. Demichev and P. Presnajder, Nucl. Phys. B 567 (2000) 360 [hep-th/9812180]; S. Minwalla, M. Van Raamsdonk and N. Seiberg, JHEP 0002 (2000) 020 [hep-th/9912072].

[7] H. Grosse and R. Wulkenhaar, Lett. Math. Phys. 71 (2005) 13 [hep-th/0403232].

[8] H. Grosse and H. Steinacker, Adv. Theor. Math. Phys. 12 (2008) 605 [hep-th/0607235]; H. Grosse and H. Steinacker, Nucl. Phys. B 707 (2005) 145 [hep-th/0407089].

[9] P. Aschieri, J. Madore, P. Manousselis and G. Zoupanos, JHEP 0404 (2004) 034 [hep-th/0310072]; P. Aschieri, J. Madore, P. Manousselis and G. Zoupanos, Fortsch. Phys. 52 (2004) 718 [hep-th/0401200].

[10] P. Forgacs and N. S. Manton, Commun. Math. Phys. 72 (1980) 15.

[11] D. Kapetanakis and G. Zoupanos, Phys. Rept. 219 (1992) 1.

[12] Y. .A. Kubyshin, I. P. Volobuev, J. M. Mourao and G. Rudolph, Lect. Notes Phys. 349 (1990) 1. 
[13] P. Aschieri, T. Grammatikopoulos, H. Steinacker and G. Zoupanos, JHEP 0609 (2006) 026 [hep-th/0606021].

[14] H. Steinacker and G. Zoupanos, JHEP 0709 (2007) 017 [arXiv:0706.0398 [hep-th]].

[15] A. Chatzistavrakidis, H. Steinacker and G. Zoupanos, Fortsch. Phys. 58 (2010) 537 [arXiv:0909.5559 [hep-th]].

[16] J. Maalampi and M. Roos, Phys. Rept. 186 (1990) 53.

[17] A. Chatzistavrakidis, H. Steinacker and G. Zoupanos, JHEP 1005 (2010) 100 [arXiv:1002.2606 [hep-th]].

[18] S. Heinemeyer, M. Mondragon and G. Zoupanos, SIGMA 6 (2010) 049 [arXiv:1001.0428 [hep-ph]].

[19] E. Ma, M. Mondragon and G. Zoupanos, JHEP 0412 (2004) 026 [hep-ph/0407236];

S. Heinemeyer, E. Ma, M. Mondragon and G. Zoupanos, AIP Conf. Proc. 1200 (2010) 568 [arXiv:0910.0501 [hep-ph]].

[20] T. Banks, W. Fischler, S. H. Shenker and L. Susskind, Phys. Rev. D 55 (1997) 5112 [hep-th/9610043].

[21] I. Chepelev, Y. Makeenko and K. Zarembo, Phys. Lett. B 400 (1997) 43 [hep-th/9701151];

A. Fayyazuddin and D. J. Smith, Mod. Phys. Lett. A 12 (1997) 1447 [hep-th/9701168];

H. Aoki, N. Ishibashi, S. Iso, H. Kawai, Y. Kitazawa and T. Tada, Nucl. Phys. B 565 (2000) 176 [hep-th/9908141].

[22] S. Iso, Y. Kimura, K. Tanaka and K. Wakatsuki, Nucl. Phys. B 604 (2001) 121 [hep-th/0101102];

Y. Kimura, Prog. Theor. Phys. 106 (2001) 445 [hep-th/0103192];

Y. Kitazawa, Nucl. Phys. B 642 (2002) 210 [hep-th/0207115].

[23] H. Aoki, S. Iso and T. Suyama, Nucl. Phys. B 634 (2002) 71 [arXiv:hep-th/0203277].

[24] S. Kachru and E. Silverstein, Phys. Rev. Lett. 80 (1998) 4855 [hep-th/9802183].

[25] E. Witten, Nucl. Phys. B 460 (1996) 335 [hep-th/9510135].

[26] G. Aldazabal, L. E. Ibanez, F. Quevedo and A. M. Uranga, JHEP 0008 (2000) 002 [hep-th/0005067].

[27] M. R. Douglas, B. R. Greene and D. R. Morrison, Nucl. Phys. B 506 (1997) 84 [hep-th/9704151].

[28] R. C. Myers, JHEP 9912 (1999) 022 [hep-th/9910053].

[29] P. Austing and J. F. Wheater, JHEP 0311 (2003) 009 [hep-th/0310170].

[30] L. E. Ibanez, JHEP 9807 (1998) 002 [hep-th/9802103].

[31] H. Steinacker, arXiv:1202.6306 [hep-th]. 